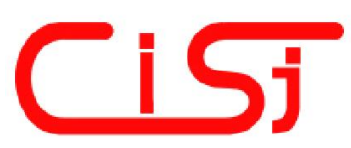

\title{
A LOW ENERGY CONSUMPTION SMART ANTENNA ADAPTIVE ARRAY SYSTEM FOR MOBILE AD HOC NETWORKS
}

\author{
Vincenzo Inzillo ${ }^{1)}$, Floriano De Rango ${ }^{1)}$, Alfonso Ariza Quintana ${ }^{2)}$ \\ 1) DIMES, University of Calabria, Via P. Bucci Rende (CS), Italy \\ v.inzillo@dimes.unical.it, derango@dimes.unical.it \\ ${ }^{2)}$ University of Malaga Av. De Cervantes, Malaga, Spain \\ aarizaq@uma.es
}

\begin{abstract}
Smart Antenna Systems (SAS) are largely employed in wireless networks for their capabilities to provide very high performances with respect to omnidirectional antennas. The use of adaptive array Smart Antenna could bring to enhance aspects such as energy management, especially in Mobile Ad Hoc Network (MANET), when the network performance closely depends on the battery life of the mobile nodes as well as on the routing protocol features. In this paper, we propose a new adaptive array Smart Antenna System that improves some Ad Hoc Distance Vector (AODV) network protocol features through a mechanism of energy consumption optimization. The system implements the Least Mean Square (LMS) as adaptive algorithm; for this purpose, we perform a co-simulation between Matlab, that implements the LMS algorithm and Omnet++, that provides a complete simulation environment for MANET. Copyright (C) Research Institute for Intelligent Computer Systems, 2017. All rights reserved.
\end{abstract}

Keywords: Beamforming; Adaptive Array; Energy Consumption; MANET; LMS; Co-Simulation; Omnet++; Matlab.

\section{INTRODUCTION}

In Mobile Ad hoc Network (MANET), energy consumption is an important issue as most mobile host operates on limited battery resources. A mobile node not only consumes its battery energy when it is actively sending or receiving packets, but it also consumes battery energy when idle and listening to the wireless medium for any possible communication requests from other nodes. Thus, energy efficient routing protocols minimize either the active communication energy which is required to transmit and receive data packets or the energy consumed during inactive periods. Generally proactive protocols consume more energy due to large routing overheads and reactive protocols suffer from route discovery latencies. In pervasive distributed environments, a very common drawback related to the energy consumption issue is that, usually, mobile nodes are equipped with omnidirectional antennas. The employment of omnidirectional antennas as part as physical layer of a node often could bring to a considerable wasting of energy, especially in cases in which a network environment requires the establishment of a lot of communication links for traffic exchange. In this case, because of the properties of omnidirectional antennas, a node that is deeply involved in communications is subject to remarkable energy consumption and consequently it could drain its battery life in a very short time, that could lead to the shutdown of the node. If such of the network nodes shutdown, the network performance especially in terms of throughput or packet delivery ratio (PDR), tend to decrease dramatically and a lot of traffic packets are lost or dropped [1-3]. A possible solution to overcome this kind of drawback is to use Smart Antenna Systems (SAS). Smart Antenna Systems are intelligent systems that considerably increase the coverage area of a communication through a hybrid multiple access technique that exploit the spatial domain. This approach is known as Space Division Multiple Access (SDMA) $[4,5]$. Smart Antenna Systems can generally be classified as either Switched-Beam or adaptive array [4]. A switched-beam system [4-7] is a system that can choose from one of many predefined patterns in order to enhance the received signal. As main property, it produces a fixed radiation pattern that remains static for all the duration of a communication. The overall goal of the switched-beam system is to increase the gain according to the location of the user. adaptive array systems $[4,8,9]$, instead, are able to direct the main beam toward a particular signal called signal of interest (SOI) minimizing the interferences that can 
occur during a communication through the execution of an adaptive algorithm that dynamically adjusts the output of the system based on the current channel conditions. In particular, the beam is conveyed toward the Angle of Arrive (AOA) or Direction of Arrive (DOA) of a certain signal that is the signal of interest. Because of their ability to produce a beam (that is electronically controlled) in a wide coverage area, adaptive array Systems greatly increase the overall capacity of a network. In this paper, we propose to design a low energy consumption adaptive array SAS technology by realizing a cosimulation that consists of two main components: a network simulator that will provide the MANET scenario and a tool which will execute the adaptive algorithm. The main purpose is to mitigate the fraction of consumed energy of nodes' battery life that is most related to the high overhead amount introduced by the routing protocol in order to limit the overall energy use only to the communications related to the intended directions also depending on the receiver position.

\section{RELATED WORKS}

There exist a considerable number of papers which deals with the adaptive array Smart Antenna Technology. Some papers highlight only on the evaluation of the adaptive algorithm to implement in SAS, such as LMS, CMA or RLS. Other surveys, instead, focus on the physical realization of an adaptive array System especially in Mobile Ad Hoc environments, where, an accurate analysis about the performance of the communications is required. In most of the recent studies about directional communications, authors try to exploit the benefits of SAS in order to realize efficient systems in terms of energy consumption. In paper [10] a new methodology is presented for enhancing the power efficiency in wireless communications by exploiting the directional features of SAS. In particular, authors make a perspective of ground with hubs by using Matlab. The authors concluded with the significance of energy efficiency with smart antennas. The paper [11] investigates about the capability of beam selection provided by SAS also suggesting the usage of this feature in order to achieve energy efficiency in wireless communication. For this purpose, authors present two schemes that consider different aspects in energy evaluation: proper energy allocation, symbol error probability and optimal selection rule in time-varying channels. A very significant paper is proposed by $\mathrm{Zhu}$ et al. [12]; authors present an energy saving mechanism called Transmission Mode (TM). The proposed method is based on a semi-SAS technology. More specifically, the mechanism attempts to achieve energy efficiency by saving the battery life of transmitting/receiving nodes without lowering the cell coverage. The process is defined by using a mechanism combined with semi-SAS technology and results demonstrated the effective robustness of the model. An analysis about the signal quality and spatial division multiplexing (SDMA) in described in [13]. This paper proposes a solution for optimizing the Signal-To-Noise-Ratio (SNR) of multiple antenna arrays in order to improve communication channel performance in an adaptively way; a measure such as SNR and dynamic range need to be used. The proposed antenna arrays are adapted and aligned in magnitude and phase. In [14] the authors perform a careful analysis about the adaptive beamforming, emphasizing the advantages in using of this strategy, making comparisons about MUSIC (Multiple Signal Classification) MVDR (Minimum Variance Distortionless Response) and ESPRIT (Estimation of Signal Parameters by Rotations Invariance Techniques) algorithm for the estimation of DOA. In [15] the authors expose the main differences between the Switched Beam and adaptive array techniques in MANET, focusing on such routing issues, when tracking position node algorithms are used. In [16] is proposed a new technique for interference suppression in WCDMA when the LMS algorithm is used, also evaluating the effects on the array factor when the number of the antenna elements changes. The paper [17] sets out a theoretical analysis about the adaptive beamforming technique is accomplished making some comparisons between the most common used adaptive algorithms. A similar analysis is performed by authors in [18], but only centering on LMS algorithm that is compared with its Normalized version (NLMS). In [19] is shown how, through the use of SAS with statistical signal processing strategies, it is possible to improve the performances in communication systems. The authors in [20] present a window based smart antenna design for MANET in which the Dynamic Source Routing (DSR) protocol is executed; this approach allows to improve also the energy consumption aspect through the minimization of the side lobes of the radiation pattern. In [21] the authors realize an adaptive array Smart Antenna System performing a co-simulation between Matlab and Opnet making some comparisons in term of performance metrics between the omnidirectional case and the adaptive array system. In particular, as adaptive algorithm is used the CMA that is implemented as Matlab Code; the code is used by Opnet, that simulates the network scenario, for the estimation of the optimum weight vector that minimizes the error in the system. In [3] is evaluated the energy consumption and the effect of mobility in MANETs in which such routing protocols like as AODV and DSR are executed; in this paper, no Smart Antenna System is implemented and the simulations are performed in NS-2. 


\section{ADAPTIVE BEAMFORMING AND ALGORITHMS}

Adaptive beamforming is a technique in which an array of antennas is exploited to achieve maximum reception in a specified direction by estimating the signal arrival from a desired direction. Basically, the adaption operation is performed by a specific algorithm whose goal is to adjust the received signals so that the desired signals are extracted once the signals are combined. The digital signal processor interprets the incoming data information, determines the complex weights (amplification and phase information) and multiplies the weights to each element output to optimize the array pattern. The optimization is based on a particular criterion, which minimizes the contribution from noise and interference while producing maximum beam gain at the desired direction. The weight vector, usually denoted as $\underline{\mathrm{W}}$, is computed using the statistics of signal $x(t)$ arriving from the antenna array. An adaptive processor will minimize the error $e(t)$ between a desired signal $d(t)$ and the array output $y(t)$. There are several adaptive beam-forming algorithms varying in complexity based on different criteria for updating and computing the optimum weights. The most used adaptive algorithms are: the Least Mean Square (LMS) algorithm, the Recursive Least Square (RLS) algorithm and the Constant Modulus algorithm (CMA). The LMS algorithm [22] was introduced by Widrow. In this algorithm, the weights are updated at every iteration by estimating the gradient of the quadratic Mean Square Error (MSE) surface, and then moving the weights in the negative direction of the gradient by a small amount, known as the step size. The convergence of this algorithm is directly proportional to the step-size parameter $\mu$. The convergence speed of LMS algorithm depends on the eigenvalues of the array correlation matrix. In a very dynamic mobile environment which yields an array correlation matrix with large eigenvalue spread, the LMS algorithm converges with a slow speed. This problem is solved with the RLS algorithm [23] by replacing the gradient step size $\mu$ with a gain matrix. The CMA adaptive beamforming [24] is a gradientbased algorithm that works on the theory that the existence of interference causes changes in the amplitude of the transmitted signal, which otherwise has a constant envelope.

\subsection{THE LEAST MEAN SQUARE (LMS) ALGORITHM}

The Least Mean Square (LMS) algorithm, is an adaptive algorithm, which uses a gradient-based method of steepest descent. LMS algorithm uses the estimates of the gradient vector from the available data. LMS incorporates an iterative procedure that makes successive corrections to the weight vector in the direction of the negative of the gradient vector which eventually leads to the minimum mean square error.

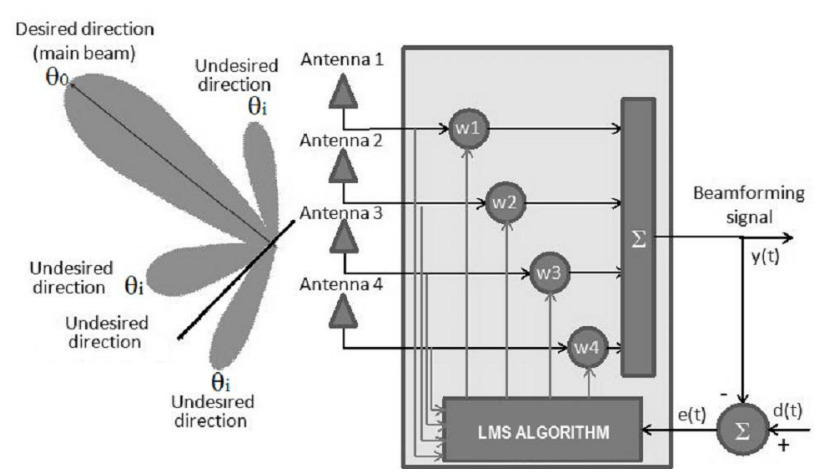

Fig. 1 - Uniform Linear Array with LMS algorithm

For example, a Uniform Linear Array (ULA) with $\mathrm{N}=4$ elements, which forms the integral part of the adaptive beamforming system, is shown in Fig. 1. The output of the antenna array is given by:

$$
x(t)=d(t) a\left(\theta_{0}\right)+\sum_{i} u_{i}(t) a\left(\theta_{i}\right)+n(t),
$$

where $d(t)$ denotes the desired signal arriving at angle $\theta_{0}$ and $u(t)$ denotes interfering signals arriving at angle of incidences $\theta_{\mathrm{i}}$ respectively. $a\left(\theta_{0}\right)$ and $a\left(\theta_{\mathrm{i}}\right)$ represents the steering vectors for the desired signal and interfering signals respectively. Therefore, it is required to construct the desired signal from the received signal amid the interfering signal and additional noise $n(t)$. The outputs of the individual elements are linearly combined after being scaled using corresponding weights such that the antenna array pattern is optimized to have maximum possible gain in the direction of the desired signal and nulls in the direction of the interferers. The steering vector is given by:

$$
a\left(\theta_{0}\right)=\left[1, e^{-j \frac{2 \pi}{\lambda} d \sin (\theta)}, \ldots ., e^{-j \frac{2 \pi}{\lambda} d[N-1] \sin (\theta)}\right],
$$

From the Method of Steepest Descent, the weight vector equation is given by:

$$
w(n+1)=w(n)+\frac{1}{2} \mu-\left[\nabla E\left\{e^{2}(n)\right\}\right]
$$

where $\mu$ is the step-size parameter and controls the convergence characteristics of the LMS algorithm while $\mathrm{n}$ is the sample size; $e^{2}(n)$ is the mean square error between the beamformer output $y(n)$ and the reference signal which is given by:

$$
e^{2}(n)=\left[d^{*}(n)-w^{H} x(n)\right]^{2},
$$


where $H$ denotes the Hermitian operator and the symbol * represents the complex conjugate operator. The gradient vector in the above weight update equation can be computed as:

$$
\nabla_{w} E\left\{e^{2}(n)\right\}=-2 r+2 R w(n)
$$

In the Method of Steepest Descent [25], the biggest problem is the computation involved in finding the values $r$ and $R$ matrices in real time. The LMS algorithm on the other hand simplifies this by using the instantaneous values of covariance matrices $r$ and $R$ instead of their actual values. Therefore, the weight update can be given by the following equation:

$$
w(n+1)=w(n)+\mu x(n) e^{*}(n),
$$

The LMS algorithm is initiated with an arbitrary value $w(0)$ for the weight vector at $n=0$. The successive corrections of the weight vector eventually lead to the minimum value of the mean squared error. The LMS algorithm converges and stay stable for $0<\mu<1 / \lambda_{\operatorname{MAX}}$ where $\lambda_{\operatorname{MAX}}$ is the largest eigenvalue of the correlation matrix $R$.

\section{MODEL IMPLEMENTATION}

Because the implementation of an adaptive algorithm directly in a network simulator is very expensive in term of time execution mainly due to the evaluation of the optimum weight vector that must be updated during all the simulation, as accomplished by authors in [18], the idea is to realize a co-simulation between a network simulator and a software that executes the adaptive algorithm. As network simulator, we chose Omnet++ (Vers. 5.0) [27], that is an extensible and modular framework, primarily used for building network environments; the main components (modules) are programmed in $\mathrm{C}++$, and are assembled into larger components and models using a high-level Network Description language (NED). As regards the adaptive algorithm we chose Matlab [28] that is a very common used numerical and graphical environment that is very suitable for mathematical algorithms execution. In our case we implemented the LMS as adaptive algorithm through three Matlab functions. For allowing the communication between the softwares we used a TCP socket-based strategy in which Omnet++ is the client and Matlab is the server side; in particular, for this purpose, we created in Omnet++ a TcpClient class that implements all functionalities to create the connection; the socket is a static variable in the class and every other module can use the socket; the class also implements the read and send functions that operate in sync during the simulation. The synchronization allows a high degree of coordination between the two sides of the socket.

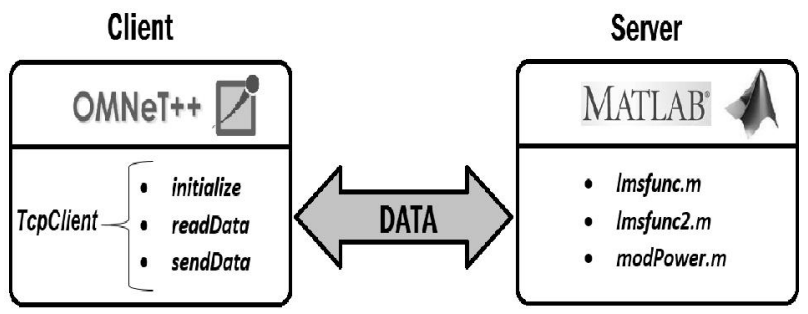

Fig. 2 - Co-Simulation model

Fig. 2 represents the communication process between the two parts. Omnet++ waits for some answers from Matlab, processes the received data and sends the new data to Matlab. This exchange process is performed until the simulation is finished. On Omnet++ side the idea is to process in RadioMedium class the communication links that occur between all senders and receivers during the simulation; in particular, a communication represents a certain signal that arrives to the receiver with a certain angle; a communication is represented by a Reception. Each Reception, that is the Desired Signal, has one or more interfering signals associated with him, that are contained in the list returned by getInterferingReceptions function. The LMS algorithm is implemented and executed by three Matlab functions: Imsfunc.m, Imsfunc2.m and modPower.m. This last function takes in input the power values of the current signal received by Omnet++ and modulates each of these values in a waveform that is passed to lmsfunc 2 and lmsfunc; these functions, firstly process the waveform of the desired and interfering signals, then execute the LMS algorithm and produce the optimum weight vector that is updated for each iteration. For allowing directional communications, nodes are equipped with the PhasedArray model created in [26]. Unlike [26], in which a switched beam array approach is realized, in this case we have a maximum radiation angle that is not static, but changes according to the adaptive algorithm operation.

Fig. 3 illustrates an example of co-simulation of the system. In particular, it can be highlighted the mobile network scenario (Fig. 3a) and the weight vector (Fig. 3b); it can be noticed how the weight vector is function of the simulation time and is clearly synchronized with the network simulator; in addition, the interfering signals are tracked by the system. 


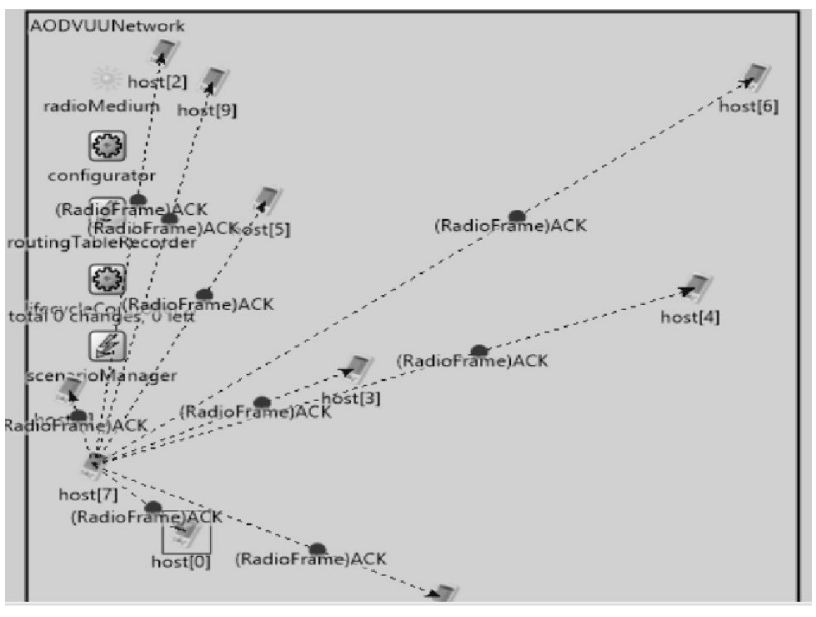

Fig. 3a-System GUI execution: network scenario

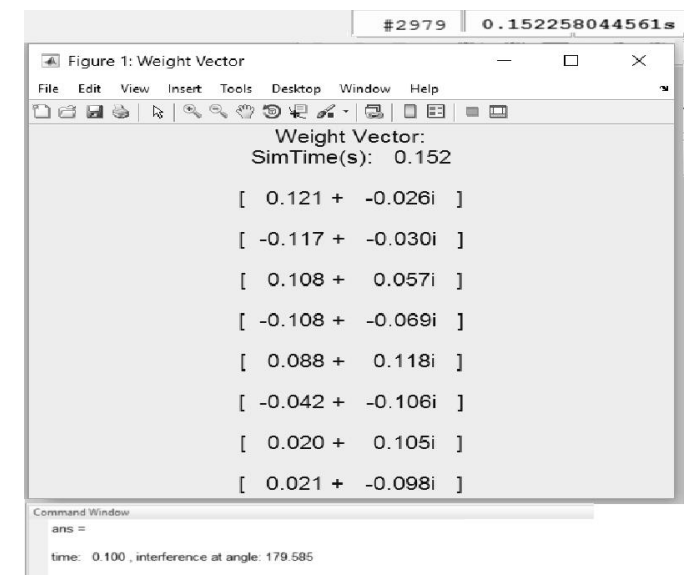

Fig. 3b - System GUI execution: weight vector computation and interference signals estimation

\section{PROPOSED ALGORITHM AND SIMULATION RESULTS}

In order to have a high accuracy in the evaluation of the results, we chose to perform 20 different simulation runs each one marked by a different seed and consider the confidence intervals produced by these runs.

The parameters used in the simulation are displayed in the Table 1 . The routing protocol used is the AODV protocol. The simulations were accomplished by varying the number of the elements of the array. The first parameter that it was evaluated is the energy consumption. For this purpose, we inserted energy simulation modules into each mobile node for allowing the emulation of the battery life behavior. The initial energy value of each node was set to 300 Joule $(\mathrm{J})$, the shutdown energy value was set to $0 \mathrm{~J}$; in this way, a node turns off when completely depletes its battery life. To enhance the performance in terms of energy consumption we developed an algorithm, that we called energy saving algorithm, that manages the switch on/off process of such elements of the array depending on the current value of nominal capacity of each node and also manages the radiation pattern based on the distance between a sender and a receiver in a communication. The algorithm was encapsulated in the antenna module in a function called switchElements(). As follows it is shown a pseudocode of the algorithm.

Table 1. Setting of parameters used in simulations

\begin{tabular}{|l|c|}
\hline $\begin{array}{l}\text { Manet Routing } \\
\text { protocol }\end{array}$ & AODV \\
\hline Antenna Type & Phased Array \\
\hline Antenna Freq. & $1 \mathrm{MHz}$ \\
\hline Distance & $0.5 \lambda$ \\
\hline Number of Nodes & 10 \\
\hline MAC Protocol & $802.11 \mathrm{~g}$ \\
\hline Simulation Area Size & $500 \times 500 \mathrm{~m}$ \\
\hline Simulation Time & $300 \mathrm{~s}$ \\
\hline
\end{tabular}

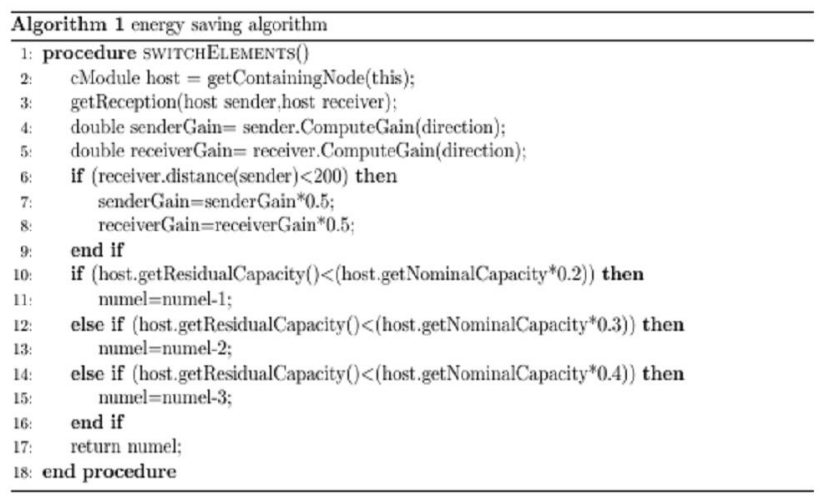

Fig. 4 - Energy saving algorithm pseudocode

Fig. 4 illustrates the energy saving procedure. The algorithm tests the current value of the battery life of each node; if this value is lower than $20 \%$ or $30 \%$ or $40 \%$ of the initial nominal energy value, it will temporally shutdown such elements of the array to reduce the energy consumption of the system. We suppose to turn off 3 array elements at most; we chose this value after several simulation tests because a greater value would reduce considerably the gain and then the Signal to Noise (SNR) during communications would become too low. After designed the energy saving algorithm we produced and evaluated the energy consumption results, firstly comparing the cases in which the algorithm is used by the system or not. Therefore, it can be noted that, if two nodes that are involved in a communication, are close together (distance $<200 \mathrm{~m}$ ), the radiation amplitude lobe is decreased by a half in order to allow a better energy distribution in the system. For each configuration, that depends on the number of elements of the array (that varies from 5 to 12 elements), we produced bar graphs in order to 
display clearly the level of residual energy. The plots are obtained by varying the simulation seed for each run, next, the collected results are averaged and finally are computed the confidence intervals. The following table illustrates the procedure for obtaining the residual capacity plot for the first configuration case (5 array elements) evaluating the confidence intervals.

Table 2. Confidence Interval evaluation example

\begin{tabular}{|c|c|c|}
\hline \multirow{2}{*}{ Run \# } & \multicolumn{2}{|c|}{ Residual Capacity (J) } \\
\cline { 2 - 3 } & $\begin{array}{c}\text { With energy } \\
\text { saving }\end{array}$ & $\begin{array}{c}\text { Without energy } \\
\text { saving }\end{array}$ \\
\hline 0 & 179,38773 & 166,51042 \\
\hline 1 & 219,20503 & 195,04767 \\
\hline 2 & 152,14002 & 142,42972 \\
\hline 3 & 196,67329 & 180,68702 \\
\hline 4 & 200,68256 & 177,49405 \\
\hline 5 & 195,03584 & 179,46922 \\
\hline 6 & 160,89811 & 145,80306 \\
\hline 7 & 175,75297 & 156,59838 \\
\hline 8 & 175,50867 & 169,74316 \\
\hline 9 & 192,06798 & 167,88182 \\
\hline 10 & 183,63345 & 172,82602 \\
\hline 11 & 166,59283 & 154,52005 \\
\hline 17 & 173,86711 & 163,46054 \\
\hline 18 & 185,68553 & 162,56762 \\
\hline 19 & 196,35018 & 171,67635 \\
\hline Average & 179,19888 & 163,84360 \\
\hline Confidence & 27,84557 & 22,20330 \\
\hline Interval up & 203,04445 & 182,04690 \\
\hline Interval down & 164,35331 & 146,74030 \\
\hline
\end{tabular}

From Tab. 2 (the entries that refer to runs from 12 to 16 are omitted) it can be observed that, all runs values are averaged and then, based on average values, confidence intervals are evaluated. This process was equally performed for all configuration cases.

The Fig. 5 shows the average residual capacity of the system for each configuration. We can note that in all cases the system wastes more energy when the algorithm is not used (case Without) compared to the case in which the algorithm is used (case With). Fig. 6 represents the average energy saving for all cases when our algorithm is used. In this figure, it can be noticed that, we have a saving that gives about from $5 \%$ (worst case) to $11 \%$ (best case). Whereas the most overall energy consumption (about $85-90 \%$ ) of the system is due to the device hardware components such as the CPU, the obtained values can be considered as acceptable values for enhancing the system performance in terms of energy consumption. We also compared the results with omnidirectional case.
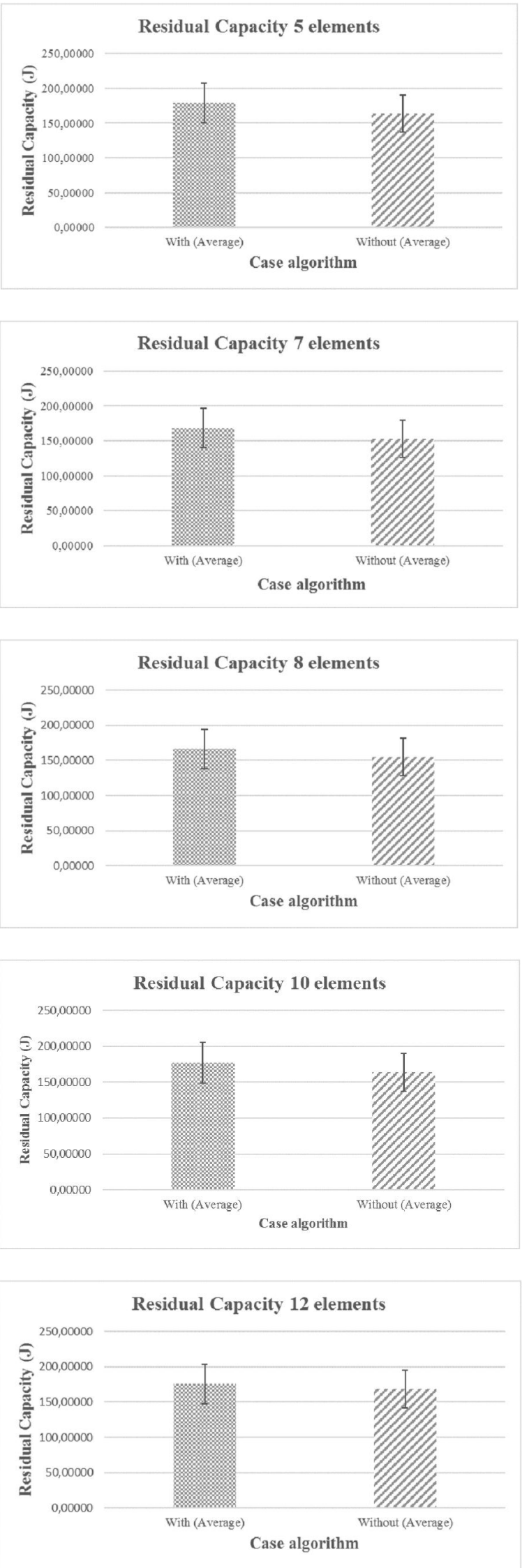

Fig. 5 - Residual Capacity with/without energy saving algorithm for all configuration cases. 


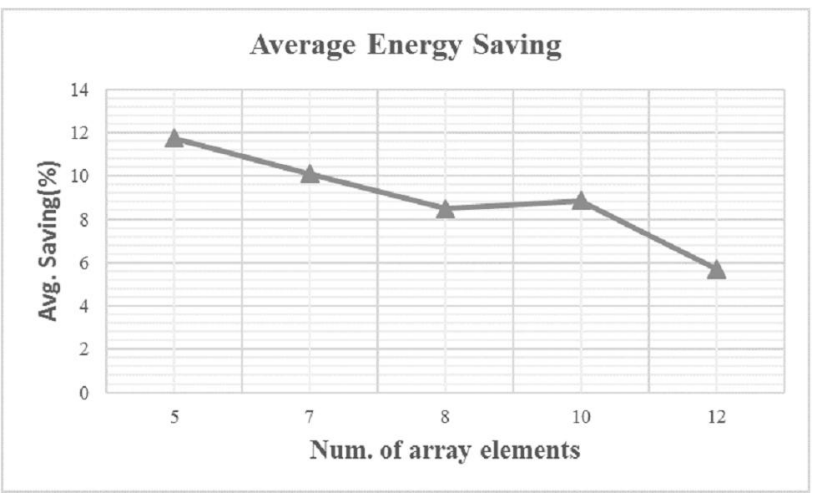

Fig. 6 - Average Saving with energy saving

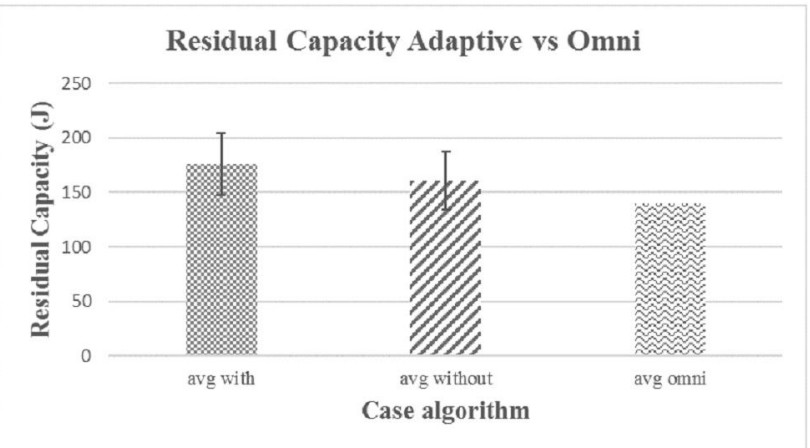

Fig. 7 - Average Residual Capacity adaptive vs omni

From Fig. 7 it can be observed that, in the omnidirectional case, mobile nodes consume a bit more energy with respect to the adaptive array system cases. In particular, this "extra" consumption gives from $15 \%$ to $25 \%$. The second parameter that it was evaluated in terms of results is the PDR that is referred to the routing protocol and both related to the energy consumption issue. As before, the first comparison was made between the case in which the energy saving algorithm is used or not; The following figure shows the average PDR of the network in the two adaptive cases:

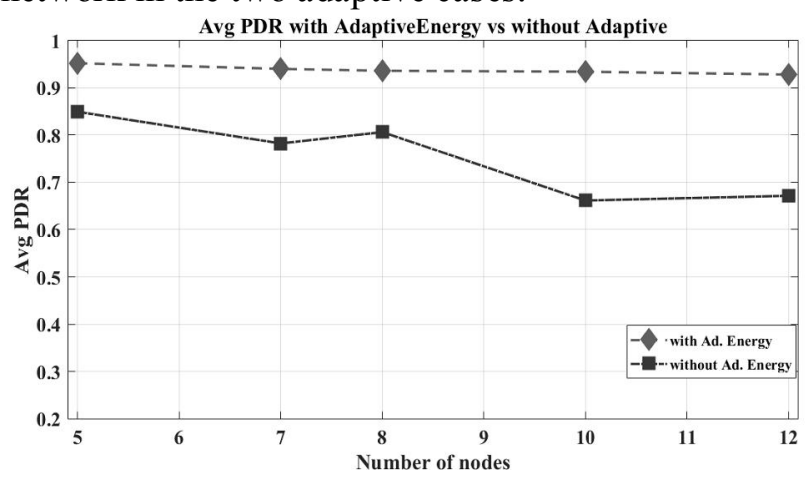

Fig. 8 - Average PDR with/without energy saving

From Fig. 8 it can be observed that, when the energy saving algorithm is used, the average PDR is really high, with a value of $93 \%$ in the worst case. It essentially remains constant as the number of the array elements increases. When the energy saving Algorithm is not used, instead, the PDR is a bit lower, and decrease significantly as the number of elements is greater than 8 . However, in the worst case, we have a value of $66 \%$, that is an acceptable value. As additional result, we compared the adaptive cases with respect to the omnidirectional case considering the worst case in which the energy saving algorithm is used and the best case in which the algorithm is not used.

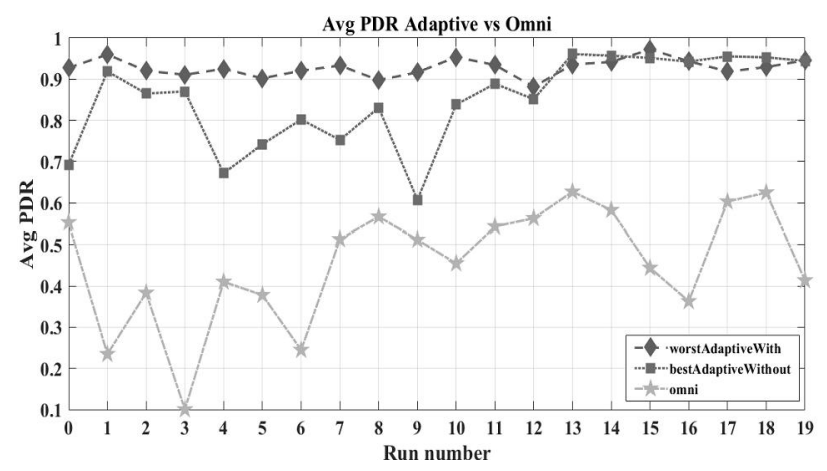

Fig. 9 - Average PDR adaptive vs omni

In this case the results are plotted in function of the simulation runs values and take in account only such configurations of the system. From Fig. 9 it can be observed that, on average, the system that uses the energy saving algorithm, also in the worst case, better performs compared to the best case in which the algorithm is not used. In the adaptive case in which the algorithm is not used we have some PDR values lower than the case in which the algorithm is used; this trend, contributes to decrease the average PDR. Numerically speaking, the average PDR when the energy saving algorithm is used is about $51 \%$ higher with respect to the omnidirectional case that registers an average PDR of about $46 \%$. Finally, when the algorithm is not used, we still obtain a considerable increase (about 39\%) compared to the omnidirectional. How it is possible to observe in the figure, the results are consistently better or equal than the case without energy saving.

\section{CONCLUSION}

We proposed an efficient adaptive array Smart Antenna System for MANETs, that also provides a mechanism for optimizing the energy consumption of the mobile nodes in the network and both enhances the global throughput in terms of Packet Delivery Ratio with respect to the case in which the mobile nodes are equipped with omnidirectional antennas. For this purpose, we developed a simple algorithm called energy saving that safeguards and monitors the battery-life of the nodes also avoiding that such network node drains its battery and 
shutdown. The algorithm avoids wastes of energy switching on/off such elements of the antenna array depending on the current level of residual capacity energy of each node and also depending on the distance between ad sender and a receiver in a communication. The system was tested making a cosimulation between the network Simulator $($ Omnet ++$)$, which provided a complete network scenario and Matlab when it was implemented the adaptive algorithm (LMS). Results, in terms of energy consumption and packet delivery ratio, shown that the system better performs when the energy saving algorithm is used compared to the case in which the algorithm is not used. More specifically, the adaptive array SAS configurations save more than $20 \%$ of node lifetime with respect to the omnidirectional case. As far as the PDR, results highlighted that, using an adaptive array system together with the energy saving mechanism, allows to improve significantly the network performance, with an average gain by more than $50 \%$ compared to the classical omnidirectional case. However, when the energy saving algorithm is not used by the system, we still have a remarkable improving of the average Packet Delivery Ratio with respect to the omnidirectional case. As future developments, we will propose to optimize the system replacing the LMS algorithm (that could result very slow in terms of computations as the number of communications increases) with a faster algorithm that provides a Variable Error Metric estimation (VMA) that we designed in [29]. Other aspect that it needs to be investigated is the node density; in particular, in order to have a precise estimation of the error introduced by interfering signals in environments in which the concentration of nodes is high, it could be necessary to implement an accurate caching mechanism; in this regard, it might result very critical to distinguish the amount of destructive power introduced mainly by the closest neighbors of a certain transmitter/receiver node, especially in contexts that changes their topology very frequently. In conclusion, our designed model should represent a valid choice for achieving good results in terms of Quality of Service (Q.o.S.) by reducing the overall error that could occur in high-noise wireless network environments while, at the same time, limiting the considerable computational burden introduced by the very expensive adaptive array SAS technology.

\section{REFERENCES}

[1] H. Xiao, D. Ibrahim, B. Christianson, "Energy consumption in mobile ad hoc networks," in Procedings of the IEEE International Wireless Communications and Networking Conference (WCNC), 2014, pp. 2599-2604.
[2] M. Palav, M. Bhosale, "Energy consumption in MANETs using energy efficient AODV protocol," Int. Journ. of Adv. Res. in Comp. Eng. and Techn. (IJARCET), vol. 3, issue 4, pp. 1463-1468, 2014.

[3] S. Kafhali, A. Haqiq, "Effect of mobility and traffic models on the energy consumption in MANET routing protocols," Int. Journ. of Soft. Comp. and Eng. (IJSCE), vol. 3, issue 1, pp. 242-249, 2013.

[4] C. Sun, X. Gao, S. Jin, M. Mattahiou, "Beam division multiple access transmission for massive MIMO communications," IEEE Trans. on Communications, vol. 63, issue 6, pp. 21702184, 2015.

[5] E. Perahia, B. Douglas, D. Pignatelli, D. Stephenson, "Spatial division multiple access for wireless networks," U.S. Patent 9131029, 2014.

[6] J. Litva, K.-Y. Lo, Digital Beamforming in Wireless Communications, Artech House, 2012.

[7] B. Sharma, T. Indranilsarkar, P. Bhattacharya, "An introduction to smart antenna system," Int. Journal of Business \& Eng. Research, vol. 8, pp. 22-26, 2014.

[8] Y. Zhou, Y. Xiaohui, "A novel adaptive beamforming algorithm for smart antenna system," in Proceedings of the Int. Conference of Computational Intelligence and Security (CIS), 2016.

[9] S. Chandran, Adaptive Antenna Arrays: Trends and Applications, Springer Science \& Business Media, 2013.

[10] M. Hanaoui, A. Mounir Rifi, "Directional Communications with Smart Antenna System to Improve Energy Efficiency in Wireless Sensor Networks," in Proceedings of the International Conference on Electrical and Information Technologies (ICEIT), Tangiers, Morocco, 4-7 May 2016.

[11] V. Kristem, N. B. Mehta, A. Molisch, "Training for antenna selection in time-varying channels," IEEE Trans. on Communications, vol. 61, issue 6, pp. 2295-2305, 2013.

[12] Y. Zhu, Z. Zeng, T. Zhang, L. Xiao, "Transmission mode selection for energy saving in the comp system with semi-smart antenna," in Proceedings of the Int. Symp. on Comm. and Infor. Techn., (ISCIT), 2012.

[13] A. S. Oluwole, V. M. Srivastava, "Analysis of smart antenna with improved signal quality and spatial processing," in Proceedings of the Progress in Electr. Research Symposium (PIERS), August 2016.

[14] R. Sahu, R. Mohan, S. Shrama, "Evaluation of adaptive beamforming algorithm of smart 
antenna," Int. Journal of Emerging Technology and Advanced Engineering, vol. 3, issue 9, pp. 592-599, 2013.

[15] S. P. Kulkarni, V. Bhavani, "Study on smart antenna systems and implementation in mobile ad hoc networks," Int. Journal of Engineering Research and Applications, vol. 4, issue 5, pp. 97-101, 2014.

[16] F. Kidane, T. Abreha, D. Mali, "Smart antenna techniques for interference suppression in WCDMA using LMS algorithm," International Journal of Computing Science and Communication Technologies, vol. 6, no. 1, pp. 916-922, July 2013.

[17] A. Arifuzzman, R. Islam, M. Tarique, "Window based smart antenna design for mobile ad hoc network routing protocol," Int. Journal of Wireless \& Mobile Networks $(I J W M N)$, vol. 7, no. 4, pp. 63-80, August 2015.

[18] M. A. Abdala, A. K. Al Zuhairy, "Integration of smart antenna systems in mobile ad hoc networks," International Journal of Machine Learning and Computing, vol. 3, no. 4, pp. 342-346, August 2013.

[19] S. Das, "Smart antenna design for wireless communication using adaptive beamforming approach," in Proceedings of the IEEE International Conference TENCON, 19-21 November 2011.

[20] N. G. R. Reddy, K. R. Devi, "Smart antennas adaptive beamforming through statistical signal processing techniques," Int. Journ. of Research in Computer and Comm. Tech., vol. 2, issue 8, pp. 400-405, 2013.

[21] S. Hossain, M. Islam, S. Serikawa, "Adaptive beamforming algorithms for smart antenna systems," in Proceedings of the Int. Conf. on Control Automation and Systems, Seoul, 2008.

[22] B. Widrow, P. E. Mantey, L. J. Griffiths, and B. B. Goode, "Adaptive antenna systems," in Proceedings of the IEEE, vol. 55, pp. 21432159, 1967.

[23] S. Haykin, Adaptive Filter Theory, Prentice Hall, 2002.

[24] M. Bhotto, A. Zulfiquar, I. Bajić, "Constant modulus blind adaptive beamforming based on unscented Kalman filtering," IEEE Signal Processing Letters, vol. 22, issue4, pp. 474478, 2015.

[25] Xu Wang, Method of Steepest Descent and its Applications, Tennessee, 2011.

[26] V. Inzillo, F. De Rango, "A directional mac approach extending Omnet++ simulator," in Proceedings of the Summer Comp. Sim. Conf. (SCSC), 2016.
[27] Omnet++ Network Simulator 5.0 Version.

[28] MATLAB, Release 2014b.

[29] V. Inzillo, F. De Rango, A. Quintana, "A new variable error metric adaptive beamforming algorithm for smart antenna systems," in Proceedings of the International Wireless Communications and Mobile Computing Conference (IWCMC), 2017.

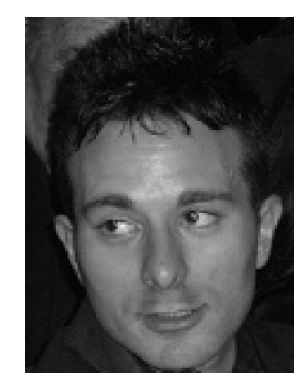

Vincenzo Inzillo is a $\mathrm{PhD}$ student of DIMES at University of Calabria, Italy. He got the master degree in Telecommunication Engineering in 2013 at University of Calabria. His topic research is about the study and the analysis of protocols for Smart Antenna Systems (SAS)

in pervasive network environments such as Mobile Ad hoc Network (MANET). He is actually involved in the development of technologies and instruments for enhancing SAS feature protocols especially regarding physical and Medium Access Control (MAC) layer.

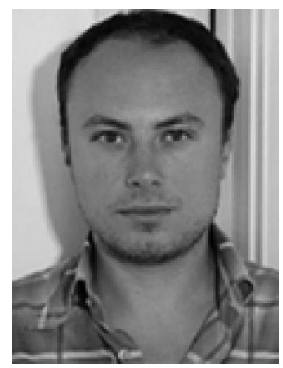

Floriano De Rango is an Assistant Professor of DIMES at University of Calabria, Italy. He received the degree in Computer Science Engineering in 2000 and a PhD in Electronics and Communications in 2005 both at the University of Calabria. From March 2004 until November 2004 he has been a Research Fellow at University of Calabria. He was recipient of Young Researcher Award in 2007. His interests include Satellite Networks, IP QOS architectures, Adaptive Wireless Networks, Ad Hoc Network, Pervasive Computing and Internet of Things (IOT).

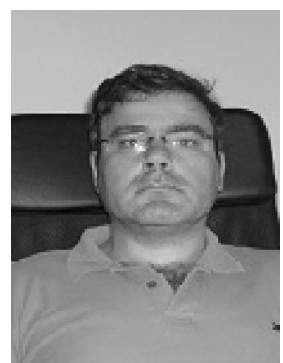

Alfonso Ariza Quintana is a Telecommunication Engineer; he got the master degree in Telecommunication Engineering at University of Malaga in 2001. He participated for several projects of public financing about cabling networks and Quality of Service (QOS) and

Wireless Network. He is very skilled in network simulation environments and communication systems; he has a very large experience about control systems and programming environments. Actually, he is Associate Professor at University of Malaga in Electronical Technologies areas. 